\title{
Emergency Thyroidectomy: A Rare Case
}

\author{
${ }^{1}$ Nitin Gupta, ${ }^{2}$ Saurabh Varshney
}

\begin{abstract}
Primary thyroid lymphoma is a rare tumor of the thyroid gland, accounting for 1 to $2 \%$ of all thyroid malignancies. The mainstay of treatment for lymphamas is chemoradiation but thyroidectomy is required for patients with large thyroid gland presenting with obstructive symptoms, such as stridor. We present here such a case of a 54-year-old female who had presented in the emergency department of our institute with a huge neck swelling and stridor. Emergency thyroidectomy was done to relieve stridor.
\end{abstract}

Keywords: Emergency thyroidectomy, Lymphoma, Malignant.

How to cite this article: Gupta N, Varshney S. Emergency Thyroidectomy: A Rare Case. Int J Otorhinolaryngol Clin 2014;6(1):47-49.

\section{Source of support: Nil}

Conflict of interest: None

\section{INTRODUCTION}

Thyroid lymphoma is a form of cancer that develops from lymphocytes. Normal lymphocytes are often present in the thyroid gland as part of a process of autoimmune inflammation that can lead to gradual destruction of tissue. ${ }^{1}$ These white blood cells may start to grow abnormally, expanding to form a mass called a lymphoma. Only about $2 \%$ of extranodal lymphomas arise within the thyroid gland, and lymphomas represent no more than $2 \%$ of all malignant thyroid tumors. ${ }^{2}$ Thyroid lymphomas can grow rapidly, and may expand to the point, where it compresses other structures in the neck, causing problems with speech, swallowing, or breathing. Primary lymphoma of the thyroid, although rare, should always be considered in the differential diagnosis of patients with thyroid nodules, goiter, and carcinomas, mainly because its prognosis and treatment differ substantially from that of the other disorders. ${ }^{3}$ Thyroid lymphoma usually responds well to treatment with external beam radiation therapy and chemotherapy. Surgical intervention is rarely required in the treatment of thyroid lymphomas. Some

\footnotetext{
${ }^{1}$ Assistant Professor, ${ }^{2}$ Professor and Head

${ }^{1}$ Department of ENT, Government Medical College and Hospital Chandigarh, India

${ }^{2}$ Department of ENT, Himalayan Institute Hospital Trust, Jolly Grant, Dehradun, Uttarakhand, India
}

Corresponding Author: Nitin Gupta, Assistant Professor Department of ENT, Government Medical College and Hospital Chandigarh, India, e-mail: nitinent123@gmail.com patients presenting with symptoms of compromised airway, dysphagia or severe pain, may require surgery to palliate their symptoms.

\section{CASE HISTORY}

A 54-year-old female presented in the emergency department with noisy breathing for the last 2 days. There was history of midline neck swelling for the last 8 years. This swelling was stationary in size initially but there was rapid progression in the size of the swelling for the last 2 months. There was history of difficulty in breathing for the last 15 days with presence of noisy breathing for the last 2 days. Hoarseness of voice was also present for the last 2 months. There was no history of dysphagia, choking or weight loss. On examination, biphasic stridor was present. A midline neck swelling of $7 \times 5 \mathrm{~cm}$ in size was present which was nontender, temperature over the swelling was normal, margins were ill-defined (Fig. 1). Swelling was diffuse and firm in consistency. It was mobile with deglutition. There was bilateral (B/L) cervical lymphadenopathy. Indirect laryngoscopy was suggestive of left vocal cord palsy.

The patient was hospitalized and put on IV fluids. Injection hydrocortisone $200 \mathrm{mg}$ IV stat was administered to the patient to relieve the stridor. A contrast enhanced CT scan of the neck was performed (Figs 2A to C). A large thyroid gland mass measuring $12 \times 10 \mathrm{~cm}$, encasing and narrowing the tracheal lumen with mediastinal extension with $\mathrm{B} / \mathrm{L}$ neck lymphadenopathy and consolidation of left upper lobe of lung. The patient was shifted to the operation theater for emergency thyroidectomy. A standard collar incision was made. The operative findings were suggestive

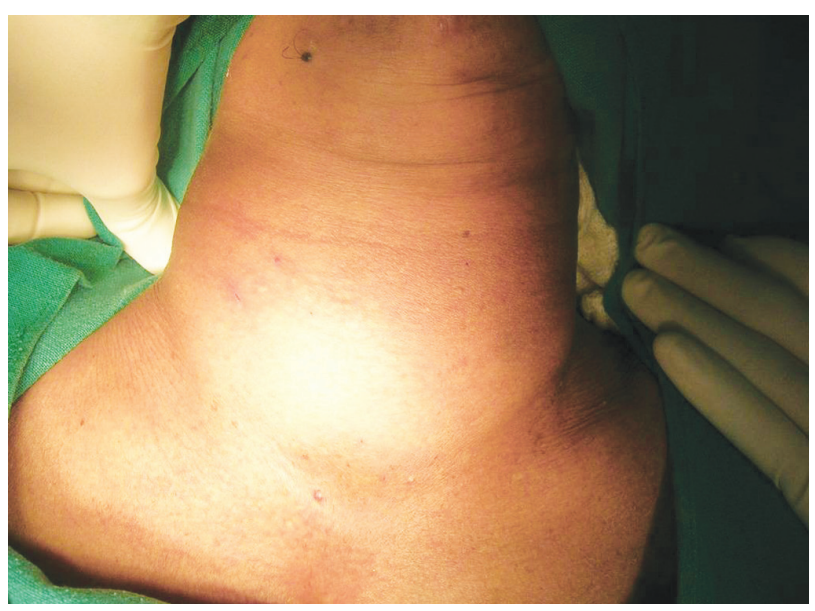

Fig. 1: A midline neck swelling of $7 \times 5 \mathrm{~cm}$ in size 


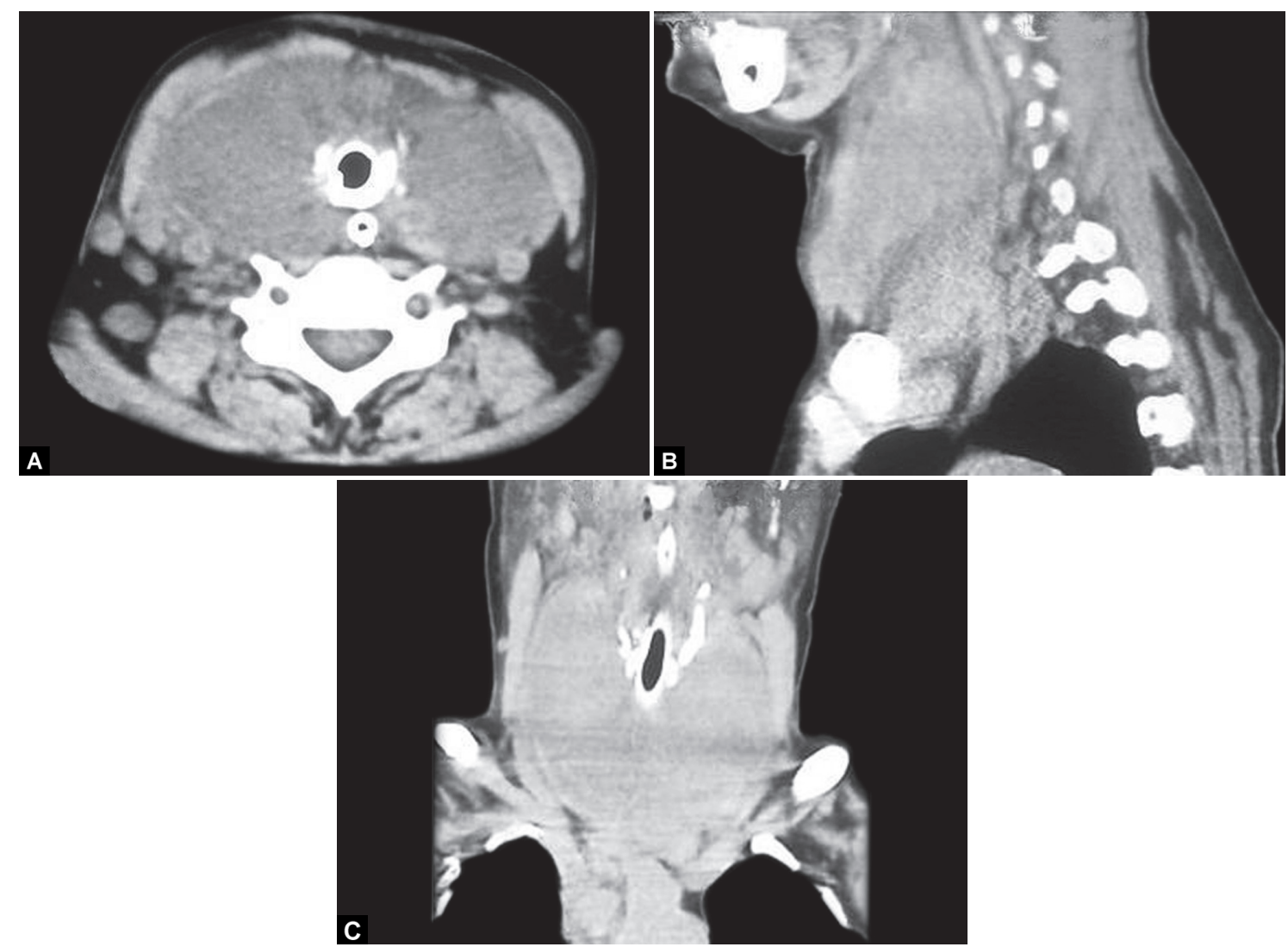

Figs $2 \mathrm{~A}$ to $\mathrm{C}$ : Contrast enhanced computed tomography neck showing a large thyroid gland mass measuring $12 \times 10 \mathrm{~cm}$, encasing and narrowing the tracheal lumen with mediastinal extension with $B / L$ neck lymphadenopathy

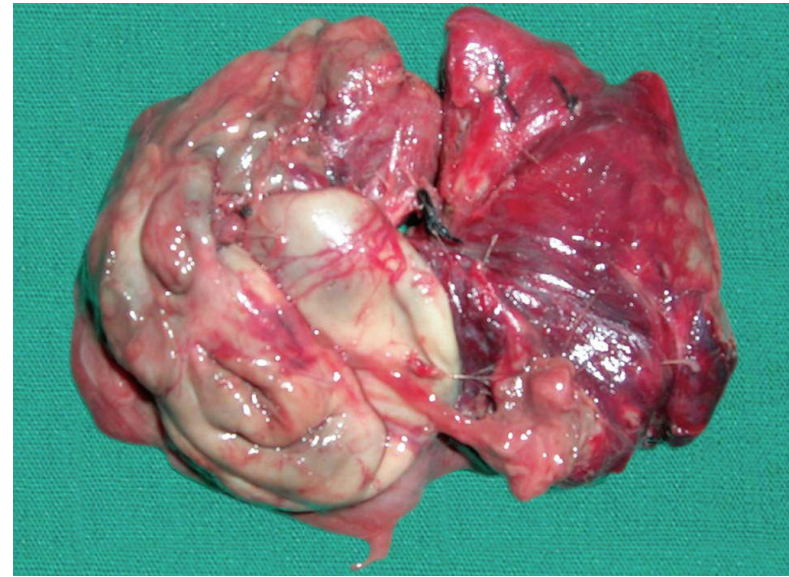

Fig. 3: Excised specimen

of enlargement of both the lobes and isthmus of the thyroid gland. The gland was going into the retrosternal region. There were multiple B/L cervical nodes. Paratracheal nodes were also enlarged. Total thyroidectomy along with neck dissection was performed (Fig. 3). Tracheostomy was also done during the surgery. The patient was shifted to the postoperative ward in a stable condition. She was put on broad spectrum antibiotics. Tracheostomy tube was removed on the 3rd postoperative day.

Histopathological examination of the removed specimen showed immunoblastic, high-grade lymphoma involving parenchyma and intrathyroid glandular tissue extending

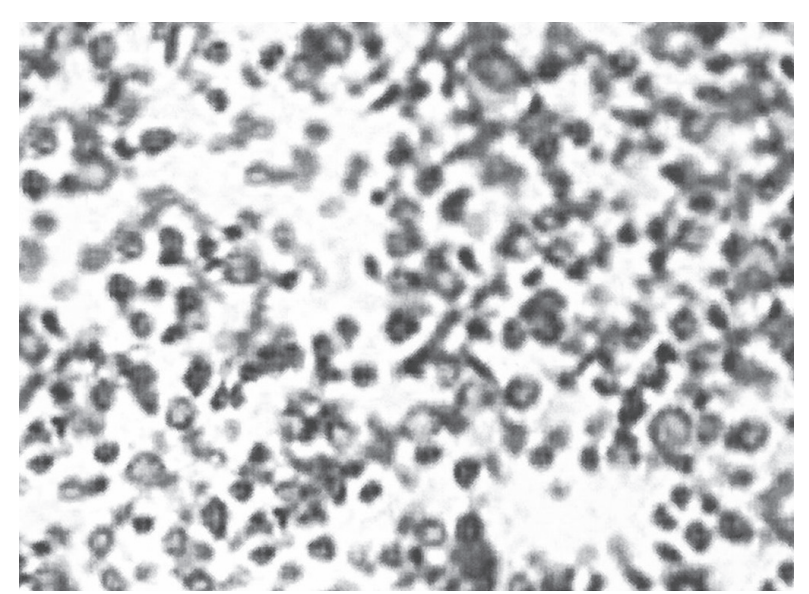

Fig. 4: Histopathological examination picture

into adipose tissue in both gland and excised nodes which was suggestive of Hashimoto's thyroiditis with malignant lymphoma (Fig. 4). A diagnosis of malignant lymphoma of the thyroid gland was made. The neck nodes also showed the features of malignant lymphoma.

The patient was given CHOP regimen of chemotherapy. Patient was kept on a regular 1 year and a month follow-up. No recurrence of the tumor was noted in the patient.

\section{Review of Literature and Discussion}

Primary thyroid lymphoma can be defined as a lymphoma that arises from the thyroid gland. This definition excludes 
lymphomas that invade the thyroid gland because of either metastasis or direct extension from an adjacent lymph node. The thyroid gland is among the most common of these extranodal sites. These lymphomas are frequently associated with Hashimoto's thyroiditis. Hypothyroidism has been observed in 30 to $40 \%$ of patients with thyroid lymphoma. ${ }^{4,5}$ Thyroid lymphomas constitute only $3 \%$ of all lymphomas and approximately $5 \%$ of all thyroid neoplasms. Primary thyroid lymphoma is a highly curable malignancy if diagnosed promptly and managed correctly. ${ }^{1,6}$ Thyroid lymphomas are more common in females with female to male ratio ranging from 2:1 to as high as 14:1 in some series. ${ }^{4}$ Thyroid lymphomas usually affect patients in 6th decade of life. The most common clinical presentation is that of a rapidly enlarging thyroid mass, frequently in association with neck adenopathy. Thyroid lymphomas usually grow faster than any other thyroid neoplasm. Hoarseness, respiratory difficulty, cough, and dysphagia can also occur as presenting symptoms. Neck adenopathy can also be present in association with the thyroid nodule. Patients undergo various investigations. Complete blood count and bone marrow are important as part of the staging evaluation. Serum lactate dehydrogenase and beta 2-microglobulin levels are important because of their usefulness in predicting the patient's prognosis. ${ }^{7}$ Thyroid function is important because of the high incidence of hypothyroidism. To evaluate the extent of the disease, radiographs of the chest and CT scans of the head and neck, chest, abdomen, and pelvis are necessary. These are critical tests for determining the stage or extent of disease. The diagnosis of thyroid lymphoma can easily be established by using fine-needle aspiration (FNA) or needle core biopsy to avoid the extensive surgery usually performed for thyroid carcinoma. ${ }^{8}$ In the past, primary thyroid large-cell lymphoma was sometimes misdiagnosed as anaplastic thyroid carcinoma. With the aid of immunophenotyping, lymphomas are easy to distinguish from thyroid carcinoma. Furthermore, the distinction between large-cell lymphoma and follicular center cell lymphoma is possible by using cytologic and immunophenotyping criteria. The most common primary thyroid lymphomas are the large-cell lymphomas. Assessment of the extent of disease in lymphomas is crucial in determining the prognosis and in selecting the treatment. The treatment of thyroid large-cell lymphoma is not different from any other lymphoma occurring in a nodal site. Treatment is based on the lymphoma subtype and the extent of disease. Most of the patients are treated with the standard $\mathrm{CHOP}$ regimen which consists of cyclophosphamide, doxorubicin, vincristine and prednisone followed by radiation therapy. The addition of rituximab provides benefit in survival and disease-free survival rates. Radiation therapy is most commonly given after 3 to 6 courses of chemotherapy followed by local irradiation. The role of surgery is only for debulking of a compressive tumor. A follow-up of the patients approximately every 3 months during the first year and every 4 months during the second year is essential. The prognosis for patients with thyroid large-cell lymphoma usually is favorable, because this condition typically presents with localized disease, which is amenable to treatment with chemotherapy and radiation. Histologically, thyroid specimens show prominent fibrosis. The fibroblastic proportion is more prominent and extra thyroid extension less pronounced. Scattered large atypical cells are embedded within the fibrotic stroma admixed with variable numbers of eosinophils, with focal nodular cellular areas. These cellular areas contain a mixed population of lymphocytes, histiocytes, plasma cells, variable numbers of eosinophils and large atypical mononuclear and multinucleated cells.

\section{CONCLUSION}

We would like to emphasize that emergency thyroidectomy is not a standard practice. Given the fact, that a similar situation may be faced by any practicing clinician at some point of time, it would be an acceptable option to discuss alternate management strategies that would target determining the cell-line of origin of the tumor, while simultaneously buying time with another option to secure the airway.

\section{REFERENCES}

1. Ansell SM, Grant CS, Habermann TM. Primary thyroid lymphoma. Semin Oncol 1999 Jun;26(3):316-323.

2. Austin JR, el-Naggar AK, Goepfert H. Thyroid cancers II. Medullary, anaplastic, lymphoma, sarcoma, squamous cell. Otolaryngol Clin North Am 1996 Aug;29(4):611-627.

3. Pasieka JL. Anaplastic cancer, lymphoma, and metastases of the thyroid gland. Surg Oncol Clin N Am 1998 Oct;7(4):707-720.

4. Tupchong L, Hughes F, Harmer CL. Primary lymphoma of the thyroid: clinical features, prognostic factors, and results of treatment. Int J Radiat Oncol Biol Phys 1986 Oct;12(10):1813-1821.

5. Velasquez WS, Jagannath S, Tucker SL, et al. Risk classification as the basis for clinical staging of diffuse large-cell lymphoma derived from 10-year survival data. Blood 1989 Aug 1;74(2): 551-557.

6. Ha CS, Shadle KM, Medeiros LJ, et al. Localized non-Hodgkin lymphoma involving the thyroid gland. Cancer 2001 Feb 15; 91(4):629-635.

7. Swan F Jr, Velasquez WS, Tucker S, et al. A new serologic staging system for large-cell lymphomas based on initial beta 2-microglobulin and lactate dehydrogenase levels. J Clin Oncol 1989 Oct; 7(10):1518-1527.

8. Pappa VI, Hussain HK, Reznek RH, et al. Role of imageguided core-needle biopsy in the management of patients with lymphoma. J Clin Oncol 1996 Sep;14(9):2427-2430. 